\title{
TITLE:
}

\section{Entanglements in quiescent and sheared polymer melts}

$\operatorname{AUTHOR}(S)$ :

Yamamoto, R; Onuki, A

CITATION:

Yamamoto, R ... [et al]. Entanglements in quiescent and sheared polymer melts. Physical Review E 2004, 70(4): 041801.

ISSUE DATE:

2004-10

URL:

http://hdl.handle.net/2433/50117

RIGHT:

Copyright 2004 American Physical Society 
PHYSICAL REVIEW E 70, 041801 (2004)

\title{
Entanglements in quiescent and sheared polymer melts
}

\author{
Ryoichi Yamamoto and Akira Onuki \\ Department of Physics, Kyoto University, Kyoto 606-8502, Japan
}

(Received 3 March 2004; published 8 October 2004)

\begin{abstract}
We visualize entanglements in polymer melts using molecular dynamics simulation. A bead at an entanglement interacts persistently for long times with the nonbonded beads (those excluding the adjacent ones in the same chain). The interaction energy of each bead with the nonbonded beads is averaged over a time interval $\tau$ much longer than microscopic times but shorter than the onset time of tube constraints $\tau_{e}$. Entanglements can then be detected as hot spots consisting of several beads with relatively large values of the time-averaged interaction energy. We next apply a shear flow with rate much faster than the disengagement motion of entangled chains. With increasing strain the chains take zigzag shapes and one-half of the hot spots become bent. The chains are first stretched as a network but, as the bends approach the chain ends, disentanglements subsequently occur, leading to stress overshoot observed experimentally.
\end{abstract}

DOI: 10.1103/PhysRevE.70.041801

PACS number(s): 61.25.Hq, 83.80.Sg, 36.20.-r

\section{INTRODUCTION}

The dynamics of dense polymer melts has been a challenging subject in current polymer physics [1-5]. While the near-equilibrium dynamics of short chains with $N<N_{e}$ can be reasonably well described by the simple Rouse model, the dynamics of very long chains with $N>N_{e}$ has not yet been well understood on the microscopic level, since it is governed by complicated entanglement effects. Here $N$ is the polymerization index or the bead number of a chain, and $N_{e}$ is the average bead number between consecutive entanglements. The reptation theory [2-8] is the most successful approach to date in describing the dynamics of entangled polymer chains in a surprisingly simple manner. It assumes that entanglements form continuous "tubes" through which the chains reptate. Its predictions have been confirmed by a number of experimental $[9,10]$ and numerical [11-21] papers, where experimentally accessible quantities such as the stress relaxation function $G(t)$, the incoherent dynamic scattering function, the mean square displacement, and the viscosity have been compared with the theoretical predictions [3]. However, $N_{e}$ estimated from the mean square displacement and that from the plateau modulus were around 30 and 70 , respectively [14], where the difference of these two estimations indicates inaccuracy of the prefactors in the predicted formulas $[3,22]$. These simulations were not direct observations of entanglements, but rather confirmed the existence of the entanglement effects indirectly using the formulas of the reptation theory. Measurements of the dynamic structure factor $S(k, t)$ by the neutron spin-echo method [9] gave information of the tube diameter $\propto N_{e}^{1 / 2}$ in the reptation theory and the resultant $N_{e}$ was consistent with the estimated value from the plateau modulus (both being of order 100).

Understanding macroscopic rheological properties of polymer melts in terms of microscopic molecular dynamics is also of great importance, where the mechanism of shearthinning behavior is very different depending on whether $N<N_{e}$ or $N>N_{e}$ and whether the glass transition is approached or not. Though still inadequate, extensive simulations have been performed in this direction [16-19,23-25].
Entanglements are discrete objects severely constraining the chain motions (only through "tubes" in the reptation theory). However, the exact nature of entanglements is still unclear and it is highly nontrivial how to detect them "directly" in simulations. Therefore, it is at present impossible to determine $N_{e}$ "precisely" from simulations. We also point out that the reptation theory does not provide the scaling functions of the physical quantities applicable even for not large $N / N_{e}$. The main aim of this paper is hence to give attempts to identify and visualize the entanglements on the microscopic level. Here we mention related simulations. Ben-Naim et al. [26] could visualize individual entanglements using the fact that incidental contacts of the particles and entanglement contacts behave differently because entanglement constraints are long lived. Gao and Weiner introduced a time-averaged atomic mobility and found that intrachain atoms of relatively low mobility tend to cluster in group along the chain for $N=200$, which suggests the existence of entanglements [15]. In the recent Kröger and Hess simulation for $10 \leqslant N \leqslant 400$ [18], the zero-shear viscosity $\eta$ (obtained at extremely weak shear) changed over from the behavior $\eta \propto N$ to the behavior $\eta \propto N^{a}$ with $a$ in the range of 3 and 3.5 around $N=N_{c} \sim 100$. This crossover polymerization index $N_{c}$ should be comparable to $N_{e}$ but they did not set $N_{c} / N_{e}=1$, probably because of the lack of the theoretical scaling formula $\eta=N f_{\text {vis }}\left(N / N_{e}\right)$ describing the Rouse-toreptation crossover as a function of $N / N_{e}$. In a similar simulation, Aoyagi and Doi [19] calculated the steady state viscosity and normal stress differences to examine nonlinear rheology for $N=100,200$, and 400. In Table I, we summarize the simulations of freely jointed bead-spring (Kremer-Grest type) chains. The estimated values of $N_{e}$ are only crude ones. Except for ours in this paper, they were obtained indirectly by fitting of numerical data to the predictions [22]. At present it is still difficult to perform sufficiently large simulations with $N \gg N_{e}$.

The organization of this paper is as follows. In Sec. II A our model system and our numerical method will be explained. In Sec. II B, in the range $N=10-250$, we will examine the time-correlation function of the end-to-end vector 
TABLE I. Summary of simulations of freely jointed chains. The second column shows the chain length in the simulations. The third and fourth columns show the system size $V^{1 / 3}$ and the simulation time $t_{\max }$ in units of the chain dimension $b N^{1 / 2}$ and the Rouse time $\tau_{0} N^{2}$. In the fifth to tenth columns the estimated values of $N_{e}$ are given (if estimated).

\begin{tabular}{|c|c|c|c|c|c|c|c|c|c|}
\hline Refs. & $N$ & $V^{1 / 3} / b N^{1 / 2}$ & $t_{\max } / \tau_{0} N^{2}$ & $N_{e}^{\mathrm{a}}$ & $N_{e}^{\mathrm{b}}$ & $N_{e}^{\mathrm{c}}$ & $N_{e}^{\mathrm{d}}$ & $N_{e}^{\mathrm{e}}$ & $N_{e}^{\mathrm{f}}$ \\
\hline KG1990 [11] & 200 & 0.9 & 1.5 & 120 & 35 & $34-50$ & 60 & & \\
\hline KLH1993 [16] & 300 & 1.5 & $(\sim 11)^{\mathrm{g}}$ & & & & & 100 & \\
\hline GW1995 [15] & 200 & 0.9 & 0.3 & & & & & & \\
\hline BGWB1996 [26] & 350 & 1.9 & 1.8 & & & & & & \\
\hline AD2000 [19] & 400 & 0.6 & 0.4 & & & & & & \\
\hline \multirow[t]{3}{*}{ PKG2000 [14] } & 350 & 1.5 & 8.2 & & & & & & \\
\hline & 700 & 1.9 & 0.2 & & $32-35$ & & & & \\
\hline & 10000 & 0.7 & 0.0002 & & $28-32$ & & $65-83$ & & \\
\hline KH2000 [18] & 400 & 2.7 & $(\sim 18)^{\mathrm{g}}$ & & & & & 100 & \\
\hline Present study & 250 & 0.7 & 80 & 100 & & & 100 & 100 & 90 \\
\hline
\end{tabular}

${ }^{a}$ From center-of-mass diffusion.

${ }^{\mathrm{b}}$ From monomer diffusion.

${ }^{\mathrm{c}}$ From scattering function.

${ }^{\mathrm{d}}$ From stress relaxation function.

${ }^{\mathrm{e}}$ From zero-shear viscosity.

${ }^{\mathrm{f}}$ From direct visualizations.

${ }^{\mathrm{g}}$ Estimated from the data given in Refs. [16] and [18].

$C(t)$, the stress relaxation function $G(t)$, the zero-shear viscosity obtained from $\eta=\int_{0}^{\infty} d t G(t)$, and the diffusion constant $D$. These data will indicate $N_{e} \sim 100$ with the aid of the reptation theory in agreement with the previous simulations. The rheological crossover from the Newtonian to shearthinning behavior will also be examined for various $N$ as in the work by Aoyagi and Doi [19]. We here stress that $G(t)$ exhibits multiscale relaxations over many decades in chain systems and its numerical calculation has been rare because it requires very long simulations $[20,21,24,25]$. We will then present time-averaging methods of detecting entanglements in quiescent states in Sec. II C and in rapidly sheared states in Sec. II D. Our "direct" observations of entanglements will again give $N_{e} \sim 100$ and enable us to examine how the stress overshoot and chain stretching occur in transient states $[6,27,28]$.

\section{NUMERICAL SECTION}

\section{A. Model}

We used the bead-spring model [11] for our polymer melts composed of $M$ chains with $N$ particles or beads in a cubic box with volume $V$. The total particle number $N M$ was 1000 for $N=10,25$, and 100, and was increased to 2500 for $N=250$. All the particle pairs interact via a truncated Lennard-Jones potential defined by [11]

$$
U_{\mathrm{LJ}}(r)=4 \epsilon\left[(\sigma / r)^{12}-(\sigma / r)^{6}\right]+\epsilon .
$$

The right-hand side is minimum at $r=2^{1 / 6} \sigma$ and the potential is truncated (or zero) for larger $r$. Using the repulsive part of the Lennard-Jones potential only in this manner, we may prevent spatial overlap of the particles [11]. The number density was fixed at $n=N M / V=1 / \sigma^{3}$, and the temperature was kept at $T=\epsilon / k_{B}$. At this temperature there was no glasslike enhancement of the structural relaxation time, but at $T$ $=0.2 \epsilon / k_{B}$ the present model with $N=10$ became glassy in our previous simulation [24]. Note that our density value is higher than the widely used value $n=0.85 / \sigma^{3}$ in the previous simulations [11,14,16-19,26]. (With increasing $n$ the free volume for particles decreases and hence $N_{e}$ in our case should be somewhat shorter than in the previous simulations.) The consecutive beads on each chain are connected by an anharmonic spring potential of the form

$$
U_{\mathrm{FENE}}(r)=-\frac{1}{2} k_{c} R_{0}^{2} \ln \left[1-\left(r / R_{0}\right)^{2}\right],
$$

where $k_{c}=30 \epsilon / \sigma^{2}$ and $R_{0}=1.5 \sigma$. The bonded pairs in the same chain thus interact via the sum of the two potentials, $U_{T}(r)=U_{\mathrm{LJ}}(r)+U_{\mathrm{FENE}}(r)$, which has a deep minimum at $r$ $=b_{\min }=0.96 \sigma$. In our simulations the actual bond lengths between two consecutive beads remained very close to $b_{\text {min }}$ with deviations being at most a few $\%$ of $b_{\min }$ even under rapid shearing. In fact, the expansion $U_{T}(r)-U_{T}\left(b_{\min }\right)$ $\cong 478 \epsilon\left(r / b_{\min }-1\right)^{2}$ follows around the minimum, so the deviation of this potential from the minimum value becomes of order $\epsilon$ even for $r-b_{\min } \sim 0.04 \sigma$. [This means that the thermal fluctuation of the bond lengths is of order $0.04 \sigma\left(k_{B} T / \epsilon\right)^{1 / 2}$.] Hereafter we will measure space and time in units of $\sigma$ and $\tau_{0}=\left(m \sigma^{2} / \epsilon\right)^{1 / 2}$, respectively, with $m$ being the particle mass, unless confusion may occur. We numerically solved Newton's equations of motion and took data after long equilibration periods of order $10^{6}\left(=5 \times 10^{8} \Delta t\right)$.

In quiescent cases, we imposed the microcanonical condition with time step $\Delta t=0.005$ under the periodic boundary condition. In the presence of shear flow, we set $\Delta t=0.0025$ and kept the temperature at $\epsilon / k_{B}$ using the Gaussian constraint thermostat to eliminate viscous heating $[29,30]$. After 


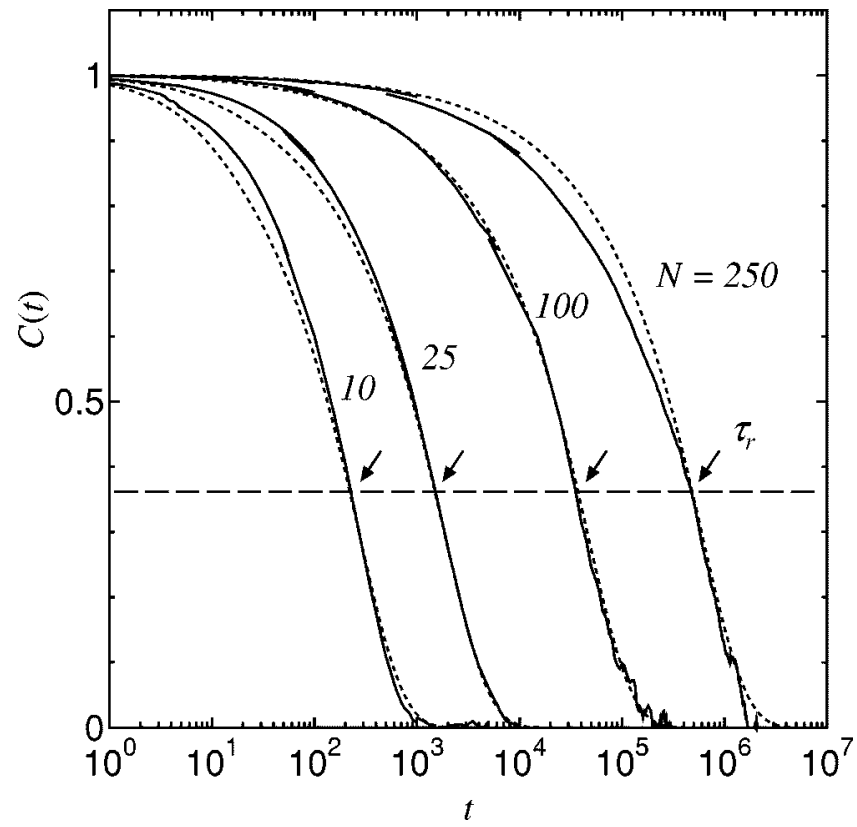

FIG. 1. The normalized time-correlation function $C(t)$ for the end-to-end vectors in Eq. (3) for various $N$ (solid lines) on a semilogarithmic scale. They can be fitted to the theoretical expression in Eq. (5) (dashed lines). The arrows indicate the terminal relaxation time $\tau_{r}$ defined by $C\left(\tau_{r}\right)=e^{-1}$, which is the Rouse relaxation time $\tau_{R}$ or the reptation time $\tau_{d}$ depending on whether $N \ll N_{e}$ or $N \gg N_{e}$.

a long equilibration period in a quiescent state in the time region $t<0$, all the particles acquired a velocity $\dot{\gamma} y$ in the $x$ direction at $t=0$, and then the Lee-Edwards boundary condition $[29,30]$ maintained the simple shear flow. The periodic boundary condition was imposed in the $x$ direction. Steady sheared states were realized after transient behavior.

For the case $N=250$ the system length $V^{1 / 3}$ is $2500^{1 / 3}$ $\cong 14$, which is of the same order as the end-to-end distance of the chains $\left(\simeq b N^{1 / 2}\right)$. For such a small system size under the periodic boundary condition, however, it is not clear how accurately we can simulate the dynamics of real entangled polymers. In future work, simulations are desirable in larger systems where both $N \gg N_{e}$ and $V^{1 / 3} \gg \sigma N^{1 / 2}$ are well satisfied.

\section{B. Crossover from Rouse to reptation dynamics}

First we show that our numerical results near equilibrium are consistent with the Rouse or reptation theory [3]. In Fig. 1 , for various $N$ on a semilogarithmic scale, we show the normalized time-correlation function of the end-to-end vector $\mathbf{P}=\mathbf{R}_{N}-\mathbf{R}_{0}$,

$$
C(t)=\left\langle\mathbf{P}\left(t+t_{0}\right) \cdot \mathbf{P}\left(t_{0}\right)\right\rangle /\left\langle\left|\mathbf{P}\left(t_{0}\right)\right|^{2}\right\rangle
$$

which is normalized such that $C(0)=1$. Here, because of the finite size of our system, the denominator and numerator on the right-hand side remain to be considerably dependent on $t_{0}$ even after taking the averages over all the chains [31]. Thus they were also averaged over the initial time $t_{0}$. The statistical (temperature-dependent) bond length $b$ is defined by

$$
\left\langle\left|\mathbf{P}\left(t_{0}\right)\right|^{2}\right\rangle=(N-1) b^{2} .
$$

In the present case $b$ is equal to $1.25 \sigma$. This value is slightly shorter than $b \simeq 1.3 \sigma$ obtained by Kremer and Grest [11] for $n=0.85 / \sigma^{3}$ but of course longer than the actual bond lengths $\left|\mathbf{b}_{j}\right|=\left|\mathbf{R}_{j+1}(t)-\mathbf{R}_{j}(t)\right| \cong b_{\min }(=0.96 \sigma)$, where $b_{\min }$ was introduced below Eq. (2) [32]. For this time-correlation function both the Rouse dynamics and the reptation dynamics predict the same simple functional form [3]. For $N \gg 1$ it is a scaling function of $t / \tau_{r}$ in terms of a relaxation time $\tau_{r}$ as

$$
C(t)=\sum_{\text {odd } p} \frac{8}{\pi^{2} p^{2}} \exp \left(-p^{2} t / \tau_{\mathrm{r}}\right)
$$

where the summation is over odd $p$ and $1 \leqslant p \leqslant N-1$. Since the first term $(p=1)$ in the summation is dominant for any $t$, this function decays nearly exponentially and $\tau_{r}$ may be determined from $C\left(\tau_{r}\right) \cong e^{-1}$. As shown in Fig. 1 , for $N$ $=10,25,100$ and 250 , we obtained

$$
\tau_{r}=270,1850,4.6 \times 10^{4}, 6 \times 10^{5}
$$

or $\tau_{r} / N^{2}=2.7,3.0,4.6$, and 9.6, respectively. The calculated curve for $N=100$ can be excellently fitted to the theoretical function $C(t)$ in Eq. (5). For the other values of $N$ the deviation is at most of order $4 \%$ around $t \sim 0.1 \tau_{r}$. However, at long times $t \gtrsim \tau_{r}$, good agreement between the calculated and theoretical curves was obtained for any $N$.

Theoretically [3], the relaxation time $\tau_{r}$ should be equal to the Rouse relaxation time

$$
\tau_{R}=\tau_{01} N^{2}
$$

for $N \ll N_{e}$ and to the reptation or disentanglement time [3]

$$
\tau_{d}=\tau_{01}^{\prime} N^{3} / N_{e}
$$

for $N \gg N_{e}$. Here $\tau_{01}=\zeta b^{2} / 3 \pi^{2} k_{B} T$ and $\tau_{01}^{\prime}=\zeta b^{2} / \pi^{2} k_{B} T$ in terms of the monomeric friction constant $\zeta$ and the bond length $b$ in Eq. (4). If $\zeta$ and $b$ are assumed to be independent of $N$, the theory predicts

$$
\tau_{01}^{\prime}=3 \tau_{01}, \quad \tau_{d} / \tau_{R}=3 N / N_{e} .
$$

It has been argued that the Rouse time $\tau_{R}$ has a well-defined physical meaning even in the reptation regime as the relaxation time of the chain contour in a tube $[3,6,7]$.

For $N=10$, the Rouse dynamics should be valid and $\tau_{r}$ $=\tau_{R}$ should hold, so our data of $b$ and $\tau_{r}$ yield

$$
\tau_{01} \cong 2.7, \quad \zeta b^{2} / k_{B} T \cong 80 .
$$

If the Rouse dynamics was assumed also for $N=25, \zeta$ was increased by $10 \%$, while a larger increase of $20 \%$ was reported for $N=10$ and $N=20$ in Ref. [11]. In this paper we used the value of $\zeta$ determined for $N=10$. Our value of $\zeta$ is about twice larger than in the previous simulations with $n \sigma^{3}=0.85$ (see Ref. [14]). We then discuss the case of $N$ $=250$, for which our data give $\tau_{r} / \tau_{R} \sim 4.6 \times 10^{4} /(0.027$ $\left.\times 250^{2}\right) \cong 3.6$ and the theoretical results in Eq. (9) give $\tau_{d} / \tau_{R}=750 / N_{e}$. In this paper (from Figs. 2, 5-7, and 10 below), we will obtain $N_{e} \sim 100$, which then yields $\tau_{d} / \tau_{R}$ $\sim 7.5$ and $\tau_{d} / \tau_{r} \sim 7.5 / 3.6 \sim 2$. Thus there arises a difference of a factor 2 between the theoretical $\tau_{d}$ and the numerically 


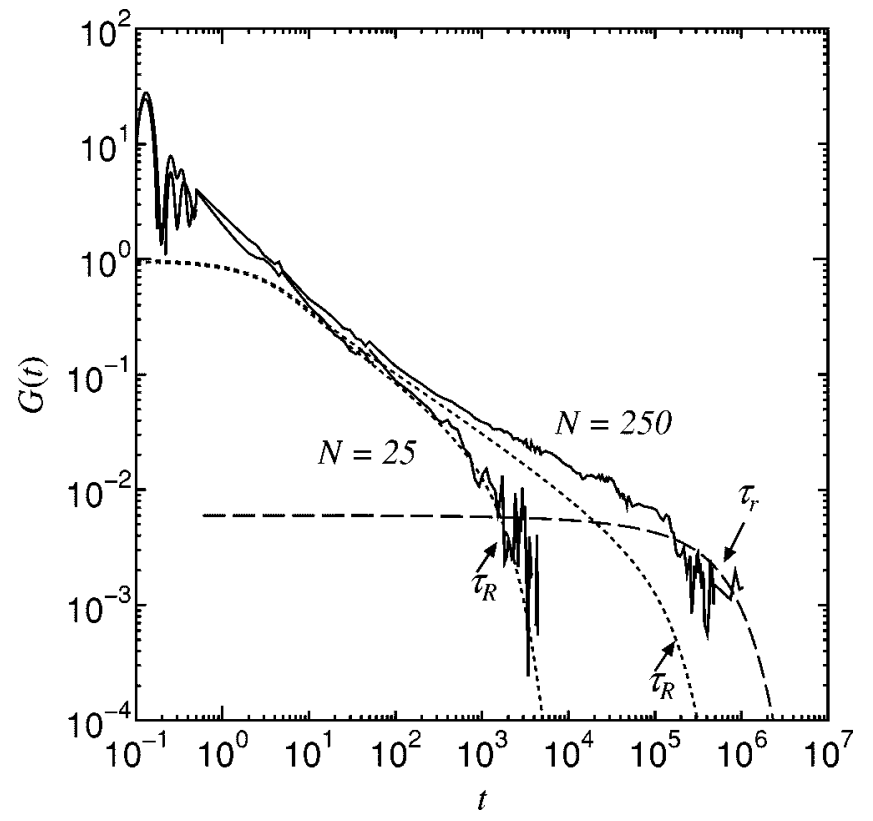

FIG. 2. The stress relaxation function $G(t)$ for $N=25$ and 250 (solid lines) in units of $\epsilon \sigma^{-3}$. Its $N$ dependence is weak for $t \lesssim 10^{2}$ but becomes increasingly stronger at later times. For $N=25$ the curve of $G(t)$ can well be fitted to the Rouse relaxation function $G_{R}(t)$ with $\tau_{01}=2.7$ and $N=25$ in Eq. (10) (left dotted line). For $N$ $=250$, it can be fitted to the reptation relaxation function $G_{\text {rep }}(t)$ in Eq. (14) for $t \gtrsim \tau_{r}$ (dashed line), where $\tau_{r}=6 \times 10^{5}$ in $C(t)$ and $N_{e}$ $=100$ in the prefactor. For comparison we also show $G_{R}(t)$ with $\tau_{01}=2.7$ and $N=250$ (right dotted line). It much deviates from the calculated curve of $G(t)$ of $N=250$ for $t \gtrsim 10^{4}$, while they agree fairly well for $10 \leqq t \leqq 10^{3}$.

obtained $\tau_{r}$. It could stem from two possible origins. One is that the Rouse-to-reptation crossover has not yet been well realized for $N=250 \sim 2.5 N_{e}$. The other is that the prefactors in the reptation theory are inaccurate as suggested by Pütz et al. for large $N$ [14]. These seem to be both relevant in the present work.

In Fig. 2, we show our numerical data of the stress relaxation function for $N=25$ and 250 expressed by

$$
G(t)=\left(k_{B} T\right)^{-1} V\left\langle\sigma_{x y}\left(t+t_{0}\right) \sigma_{x y}\left(t_{0}\right)\right\rangle
$$

where $V$ is the system volume and the tensor $\sigma_{\alpha \beta}(t)$ is defined by $[16,17,24]$

$$
V^{-1} \int d \mathbf{r} \Pi_{\alpha \beta}(\mathbf{r}, t)=p(t) \delta_{\alpha \beta}-\sigma_{\alpha \beta}(t)
$$

in terms of the microscopic stress tensor $\Pi_{\alpha \beta}(\mathbf{r}, t)$. The pressure $p(t)$ may be defined such that $\sigma_{\alpha \beta}(t)$ is traceless or deviatoric. See Refs. [16,17,24] for the microscopic expression of $\sigma_{\alpha \beta}(t)$. The curve for the short chain case $N=25$ and $M=40$ is a result of the averages over 10 independent runs and over the initial time $t_{0}$. For $t \gtrsim 10$ it can be excellently fitted to the Rouse relaxation function (left dotted line) [3],

$$
G_{R}(t)=\frac{n k_{B} T}{N} \sum_{p=1}^{N-1} \exp \left(-p^{2} t / \tau_{01} N^{2}\right),
$$

where $\tau_{01}=2.7$ as determined in Eq. (10). However, for the longest chain case $N=250$ and $M=10, G(t)$ was calculated from a single very long run performed up to $t=5 \times 10^{6}$ $=10^{9} \Delta t$ with the average over $t_{0}$ being taken. For $10 \leqq t$ $\lesssim 10^{3}$, it agrees with $G_{R}(t)$ (right dotted line), but in the terminal time range $t \gtrsim \tau_{r}=6 \times 10^{5}$ the calculated $G(t)$ relaxes much slower than predicted by the Rouse dynamics. In Fig. 2, although the characteristic plateau behavior for $N$ $\gg N_{e}$ is not clearly seen, we draw the theoretical stress relaxation function $G_{\text {rep }}(t)$ given by [3]

$$
G_{\text {rep }}(t)=\frac{3 n k_{B} T}{5 N_{e}} C(t)
$$

with $N_{e}=100$ (dashed line), where $C(t)$ is defined in Eq. (5). The agreement appears to be fair for $t \gtrsim 10^{5}$, but due to the noisy behavior and the absence of well-defined plateau in $G(t)$, we may claim only that $N_{e}$ is in the range $70 \leqq N_{e}$ $\lesssim 150$ from the fitting. Note that the value of $\tau_{r}$ at $N=250$ used for $C(t)$ in Eq. (14) is obtained from Fig. 1 or Eq. (6).

Further remarks regarding Fig. 2 are as follows. (i) First, the initial value $G(0)=V\left\langle\sigma_{x y}^{2}\right\rangle / k_{B} T$ takes a very large value about 92 (not seen in Fig. 2) and is nearly independent of $N$. Because the initial values of $G_{R}(t)$ and $G_{\text {rep }}(t)$ are much smaller as $G_{R}(0) \cong n k_{B} T \sim 1$ and $G_{\text {rep }}(0) \cong 3 n k_{B} T / 5 N_{e}$ $\sim 0.25$, agreement of $G(t)$ with these model relaxation functions is attained only after transient relaxations [33]. As reported previously [20,24], the high-frequency vibration of the bond lengths (with period 0.14 here) gives rise to initial oscillatory behavior in $G(t)$ at short times $(t \lesssim 1)$ [34]. (ii) Second, we notice marked noisy behavior of the curves in Fig. 2 for $t \gtrsim t_{r}$ as already reported in Refs. [20,24]. For such large time separation, the correlation of order $10^{-4}$ of the initial value needs to be picked up, while the amplitude of the thermal fluctuations of $\sigma_{x y}(t)$ at each time $t$ is given by

$$
\sigma_{\mathrm{fl}}=\left[\left\langle\sigma_{x y}(t)^{2}\right\rangle\right]^{1 / 2} \sim\left[G(0) k_{B} T / V\right]^{1 / 2} .
$$

This quantity is of order $(92 / 2500)^{1 / 2} \sim 0.2$ for the case of $N=250$ in the dimensionless units [24]. The noisy behavior in $G(t)$ in the terminal time range can in principle be eliminated only if the system size is very large and/or many runs are performed.

In Fig. 3, we summarize our results of the relaxation time $\tau_{r}$, the zero-frequency shear viscosity $\eta=\int_{0}^{\infty} d t G(t)$ in the linear regime, and the diffusion constant $D$, as functions of $N$. Here $\eta \propto N$ in the Rouse dynamics and $\eta \propto N^{3} / N_{e}^{2}$ in the reptation dynamics. The diffusion constant was obtained from the relation

$$
\left\langle\left|\Delta \mathbf{R}_{G}(t)\right|^{2}\right\rangle=6 D t,
$$

where $t>\tau_{r}$ and $\Delta \mathbf{R}_{G}(t)=\mathbf{R}_{G}\left(t_{0}+t\right)-\mathbf{R}_{G}\left(t_{0}\right)$ is the displacement vector of the center of mass of the chains in a time interval $\left[t_{0}, t+t_{0}\right]$ with width $t$. At the end of the runs, the mean square displacement much exceeds $\left\langle\left|P\left(t_{0}\right)\right|^{2}\right\rangle$ in Eq. (4) for $N \leqslant 100$, but is only 1.5 times larger for 


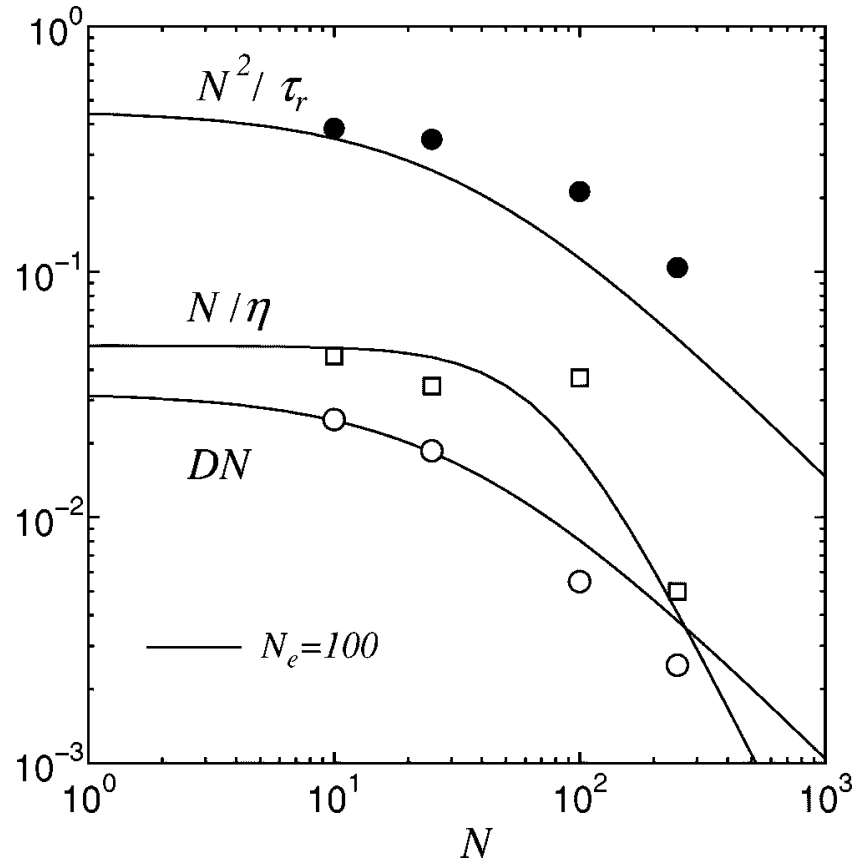

FIG. 3. $N$ dependence of $N^{2} / \tau_{r}(\bullet), N / \eta(\boldsymbol{\square})$, and $N D(\circ)$. These quantities are expected to tend to the Rouse limits for 1 $\ll N \ll N_{e}$. The solid lines represent $\left(3 \pi^{2} k_{B} T / \zeta b^{2}\right) /\left(1+3 N / N_{e}\right)$, $\left(36 / n \zeta b^{2}\right) /\left(1+8 N^{2} / 5 N_{e}^{2}\right)$, and $\left(k_{B} T / \zeta\right) /\left(1+3 N / N_{e}\right)$ with $N_{e}=100$. These approximate expressions extrapolate the predicted formulaes of the Rouse and reptation behaviors in the two limits $N \ll N_{e}$ and $N \gg N_{e}$.

$N=250$. It is known that $D \propto N^{-1}$ in the Rouse dynamics and $D \propto N_{e} / N^{2}$ in the reptation dynamics [3]. In Fig. 3 we can see that the dynamics of our system cannot be described by the Rouse dynamics with increasing $N(\gtrsim 100)$. Though the largest $N(=250)$ is only 2.5 times larger than our estimated $N_{e}$, the $N$ dependencies in Fig. 3 are consistent with the crossover from the Rouse to reptation dynamics reported in the previous simulations [11-14,16-18].

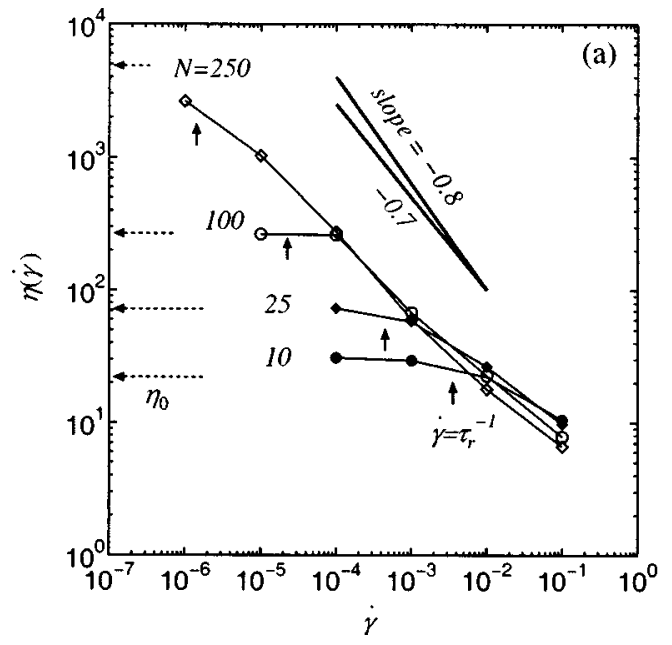

We also show that our model polymer melts exhibit strongly nonlinear behavior for $\dot{\gamma}>\tau_{\mathrm{r}}^{-1}$. Figure 4 displays the steady-state shear viscosity $\eta$ in (a) and the steady-state normal-stress difference $N_{1}$ in (b), where

$$
\eta(\dot{\gamma})=\left\langle\sigma_{x y}\right\rangle / \dot{\gamma}, \quad N_{1}(\dot{\gamma})=\left\langle\sigma_{x x}\right\rangle-\left\langle\sigma_{y y}\right\rangle .
$$

Here the stress components consist of the contributions from all the particles as defined by Eq. (12) and we took the time average $\left\langle\sigma_{i j}\left(t_{0}\right)\right\rangle$ over $t_{0}$ within an interval with width 200 . The vertical (solid) arrows indicate the points at which $\dot{\gamma}$ $=\tau_{r}^{-1}$. For $\dot{\gamma}<\tau_{r}^{-1}$, the system shows Newtonian behavior, and $\eta$ converges to the zero shear viscosity, $\eta=\int_{0}^{\infty} d t G(t)$, indicated with the horizontal (dotted) arrows in Fig. 4(a). On the other hand, $\eta(\dot{\gamma})$ at high shear is nearly independent of $N$ in agreement with rheology experiments [1,7,35] and the previous simulations [16-19], while $N_{1}(\dot{\gamma})$ is an increasing function of $N$ at any shear rate. If the data of $N=100$ and 250 at high shear are fitted to power laws, we roughly obtain $\eta(\dot{\gamma}) \sim \dot{\gamma}^{-a}$ and $N_{1}(\dot{\gamma}) \sim \dot{\gamma}^{a_{1}} N^{c_{1}}$ with $a \sim 0.7, a_{1} \sim 0.5$, and $c_{1} \sim 1.0$. The second normal stress difference $N_{2}(\dot{\gamma})=\left\langle\sigma_{y y}\right\rangle$ $-\left\langle\sigma_{z z}\right\rangle$ (not shown here) was smaller than $N_{1}(\dot{\gamma})$ by about two orders of magnitude at any shear rates. In the literature $[3,6,7]$, it has been argued that the relaxation of the chain contours occurs on the time scale of $\tau_{R}$ and is of crucial importance in nonlinear rheology under rapid deformations. In our case, however, Fig. 1 shows that the ratio $\tau_{r} / \tau_{R}$ is only about 3.6 even for $N=250$, so we cannot draw definite conclusions on the overall nonlinear rheology.

\section{Entanglements in quiescent states}

For the longest chain case $N=250$, we attempt to identify and visualize entanglements. We expect that there should be singular enhancement in the Lennard-Jones potential energy between particles near an entanglement point. To examine this effect, we first define the potential energy of nonbonded interaction on the $i$ th particle by

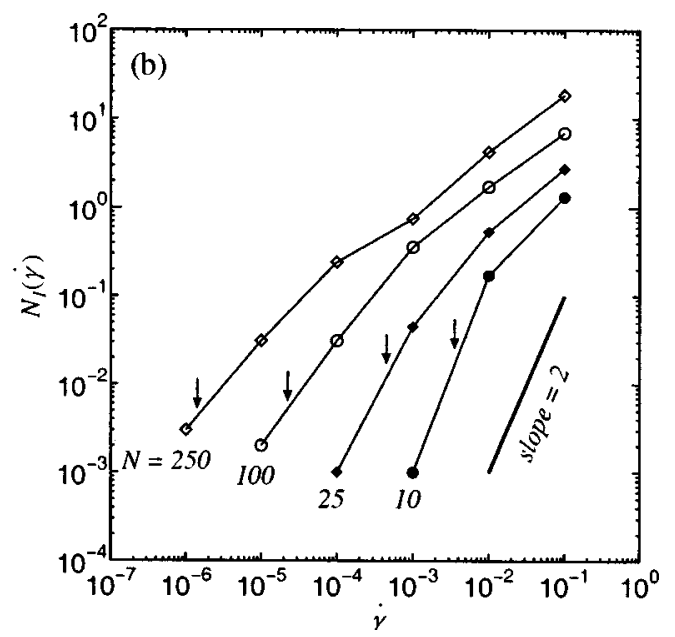

FIG. 4. Calculated viscosity $\eta(\dot{\gamma})=\sigma_{x y} / \dot{\gamma}$ in (a), and normal stress difference $N_{1}(\dot{\gamma})=\sigma_{x x}-\sigma_{y y}$ in (b) in steady states in shear for various $N$. The vertical (solid) arrows indicate the points at which $\dot{\gamma}=\tau_{r}^{-1}$, and the horizontal (dotted) arrows indicate the zero-shear viscosity $\eta$ $=\int_{0}^{\infty} d t G(t)$ plotted in Fig. 3. As guides for the eyes, we draw straight lines of slope -0.7 and -0.8 in (a) and a line of slope 2 in (b). Note that $N_{1}(\dot{\gamma}) \propto \dot{\gamma}^{2}$ for Newtonian shear. 


$$
W_{i}(t)=\sum_{j \in \text { nonbond }} U_{\mathrm{LJ}}\left(\left|\mathbf{r}_{i}(t)-\mathbf{r}_{j}(t)\right|\right) .
$$

The particle pairs $i$ and $j$ mostly belong to different chains, giving rise to the interchain contributions in Eq. (18). However, we also include the contributions from the pairs belonging to the same chain but being not adjacent to each other $(j \neq i \pm 1)$. We found that the thermal fluctuations of $W_{i}(t)$ are so large that their distributions are nearly Gaussian at each time $t$ without any noticeable correlations even between adjacent beads ( $i$ and $i+1$ on the same chain). The nonbonded interactions $W_{i}(t)$ consist mostly of rapidly varying thermal fluctuations uncorrelated to one another. With the end of reducing such rapid components, we introduce the time average of $W_{i}(t)$,

$$
W_{i}(t, \tau)=\frac{1}{\tau} \int_{0}^{\tau} d t^{\prime} W_{i}\left(t+t^{\prime}\right),
$$

where the time interval $\tau$ is much longer than the microscopic time $\tau_{0}$ (=1 in our units). This time average is analogous to that introduced by Thirumalai and Mountain in analyzing dynamics of supercooled liquids [36]. We introduce the variance $\sigma(\tau)$ [36],

$\sigma(\tau)^{2}=\frac{1}{M N} \sum_{i}\left[W_{i}\left(t_{0}, \tau\right)-\langle W\rangle\right]^{2}=\frac{2}{\tau} \int_{0}^{\tau} d t^{\prime}\left(1-t^{\prime} / \tau\right) F^{N B}\left(t^{\prime}\right)$,

where

$$
F^{N B}\left(t^{\prime}\right)=\left\langle W_{i}\left(t_{0}+t^{\prime}\right) W_{i}\left(t_{0}\right)\right\rangle-\langle W\rangle^{2} .
$$

Here $\langle W\rangle$ is the thermal average of $W_{i}\left(t_{0}\right)$ over all the beads nearly independent of $i$ and $t_{0}$. In Eq. (20), the timecorrelation function $F^{N B}\left(t^{\prime}\right)$ is assumed to be independent of the initial time $t_{0}$. We found that $\sigma(\tau)^{2}$ decays nearly as $A \tau^{-1}$ for $\tau \gg 1$. The coefficient $A$ should then be given by $A$ $=2 \int_{0}^{\infty} d t^{\prime} F^{N B}\left(t^{\prime}\right)$. This indicates that most of the contributions in the time integral in Eq. (19) behave as thermal white noise [36].

From the reptation theory $[3,9]$ the distance of the thermal bead motions during a time interval of $\tau$ is estimated in the short-time range $\tau \ll \tau_{e}$ as

$$
\ell(\tau)=\sqrt{\left\langle|\Delta \mathbf{R}(\tau)|^{2}\right\rangle} \sim a\left(\tau / \tau_{e}\right)^{1 / 4} \sim \tau^{1 / 4},
$$

where $\Delta \mathbf{R}(\tau)=\mathbf{R}\left(t_{0}+\tau\right)-\mathbf{R}\left(t_{0}\right)$ is the displacement vector of a bead during a time interval of $\tau, a=b N_{e}^{1 / 2}$ is the tube diameter, and [37]

$$
\tau_{e} \sim \tau_{01} N_{e}^{2} \sim 3 \times 10^{4}
$$

is the onset time of the effect of tube constraints. The relation in Eq. (22) is well satisfied in the range $10<\tau<10^{4}$ for $N$ $=250$ in our simulation. This power law has been confirmed in the previous simulations $[11,12,14]$. To achieve visualization of entanglements in the following, we should require $\ell<a \sim N_{e}^{1 / 2}$ and hence $\tau<\tau_{e} \sim N_{e}^{2}$ in Eq. (19).

With the above time-averaging procedure, the white noise should be mostly eliminated with increasing $\tau$ and, as a result, long-lived correlations due to a small number of
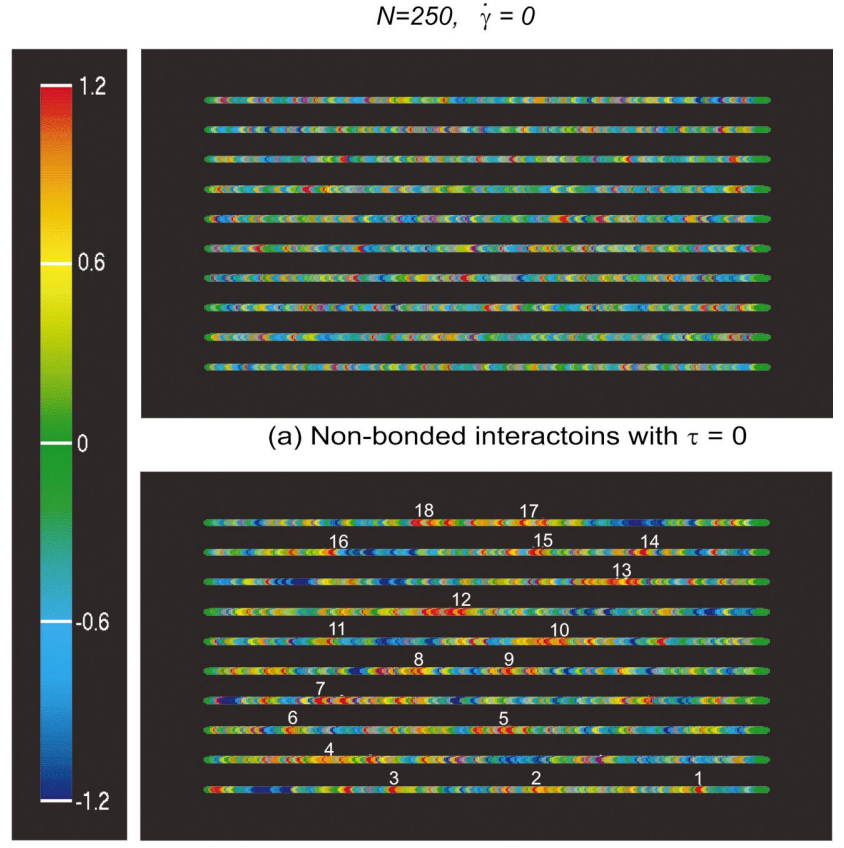

(a) Non-bonded interactoins with $\tau=0$

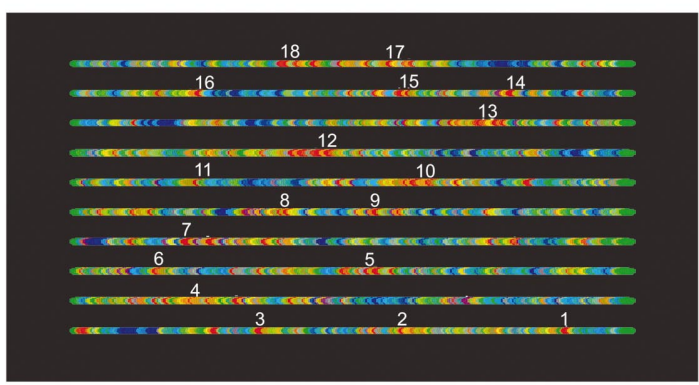

(b) Non-bonded interactions with $\tau=5000$

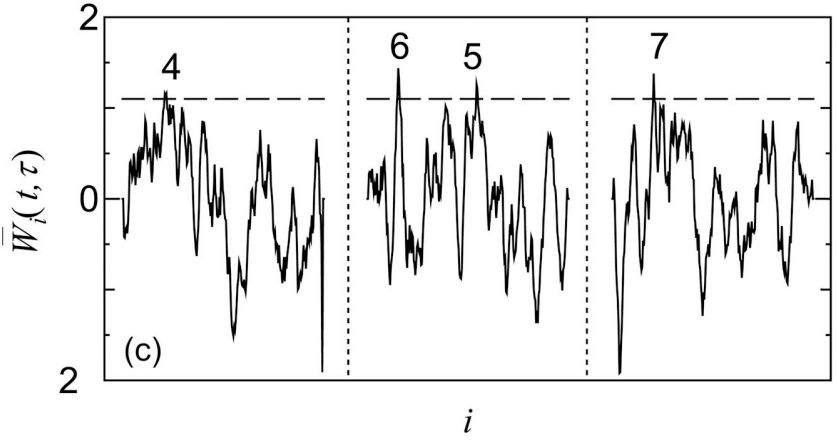

FIG. 5. (Color) Distributions of the nonbonded interaction energy on the chains for $N=250$ in a quiescent state. The 10 chains are straightened on the plane. In (a) the normalized values $\left[W_{i}(t)\right.$ $-\langle W\rangle] / \sigma(0)$ are shown, where $\tau=0$ and no correlations along the chains can be seen. In (b) the normalized, time-averaged values $\left[W_{i}(t, \tau)-\langle W\rangle\right] / \sigma(\tau)$ with $\tau=5 \times 10^{3}$ are shown, which are distinctly large in line segments consisting of several beads (in orange) presumably due to entanglements. In (b) the active spots are numbered from 1 to 18 according to the criterion given around Eq. (24). In (c) the data of $\left[W_{i}(t, \tau)-\langle W\rangle\right] / \sigma(\tau)$ for the three chains with the spots 4-7 in (b) are shown. The horizontal axis denotes the bead numbers $1 \leqslant i \leqslant 250$ for the three chains. The beads above the broken line are defined as "active" beads.

entanglements should become detectable in the range 1 $\ll \tau<\tau_{e}$. In order to demonstrate this, in Fig. 5, we display normalized instantaneous values $\left[W_{i}(t)-\langle W\rangle\right] / \sigma(0)$ in (a) and normalized time-averaged values $\left[W_{i}(t, \tau)-\langle W\rangle\right] / \sigma(\tau)$ for $\tau=5 \times 10^{3}=0.8 \times 10^{-2} \tau_{r} \sim 0.2 \tau_{e}$ in (b) at an appropriate time $t$ after a long equilibration period. For this $\tau$, the distance $\ell(\tau)$ in Eq. (22) is given by 4.5 . Here the 10 chains in our system at time $t$ are straightened horizontally for the visualization purpose. Similar pictures of a time-averaged atomic mobility were given by Gao and Weiner [15], where the presence of low mobility clusters was assumed to be due to entanglements. In (a) almost no correlation can be seen 

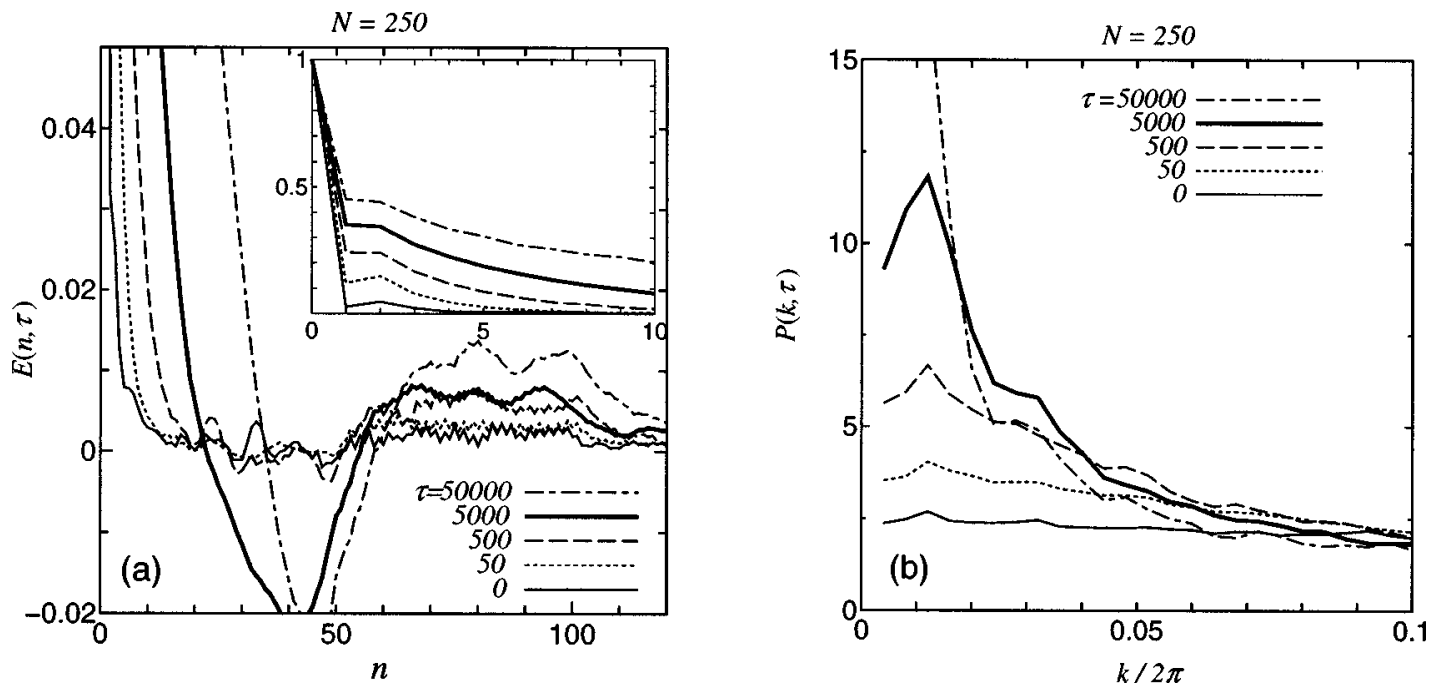

FIG. 6. The intrachain correlation function $E(n, \tau)$ of the nonbonded interactions defined by Eq. (25) in (a), and its Fourier transformation $P(k, \tau)$ defined by Eq. (26) in (b), for $N=250$. Here $\tau=0$ (thin-solid line), $5 \times 10$ (dotted line), $5 \times 10^{2}$ (dashed line), $5 \times 10^{3}$ (bold line), and $5 \times 10^{4}$ (dotted-dashed line). With increasing $\tau$ we can see development of the minimum and the maxima presumably due to entanglements. The inset in (a) shows $E(n, \tau)$ for small $n$ for these $\tau$.

$$
N=250, \quad \dot{\gamma}=0.001
$$

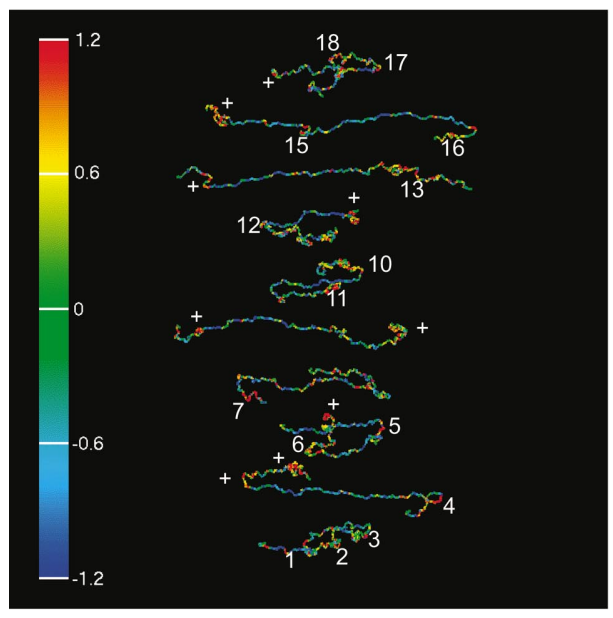

(a) $\dot{\gamma} t=5$

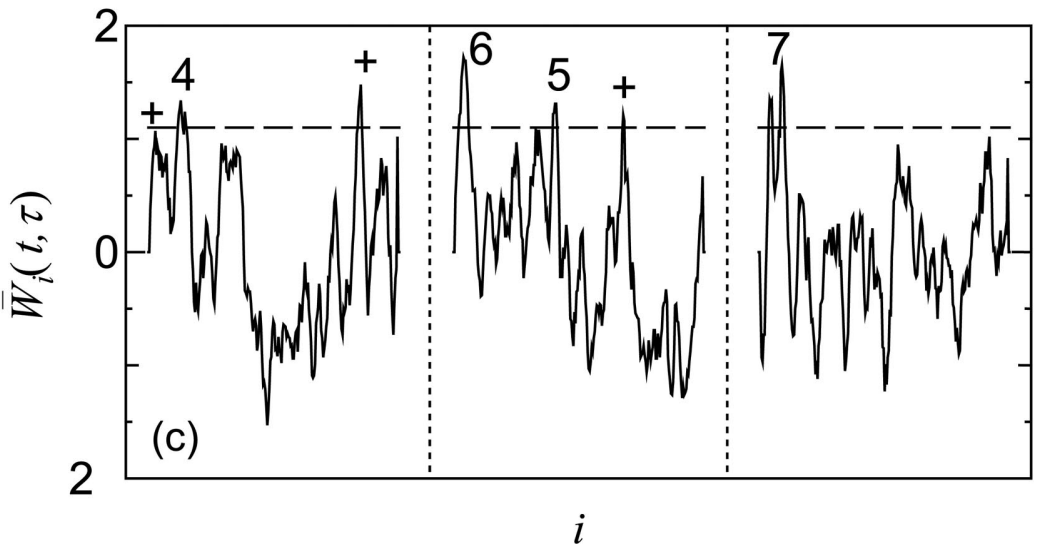

FIG. 7. (Color) Snapshots of the deformed chains with $N=250$ on the $x z$ plane at $\dot{\gamma} t=5$ in (a) and at $\dot{\gamma} t=10$ in (b), where a shear flow with $\dot{\gamma}=10^{-3}$ was applied at $t=0$ with the same initial chain configuration as in Fig. 5 (b). The nonbonded interactions with $\tau$ $=500$ are written on the chains. The active spots satisfying the criterion given around Eq. (24) are detected, among which the numbered segments correspond to those in Fig. 5(b). However, the active spots which do not correspond to those in Fig. 5(b) are marked by + . The flow is in the horizontal $(x)$ direction, and the shear gradient is in the out-ofplane $(y)$ direction. In (c) the data of $\left[W_{i}(t, \tau)-\langle W\rangle\right] / \sigma(\tau)$ for the three chains with the spots $4-7$ in (b) are shown. 


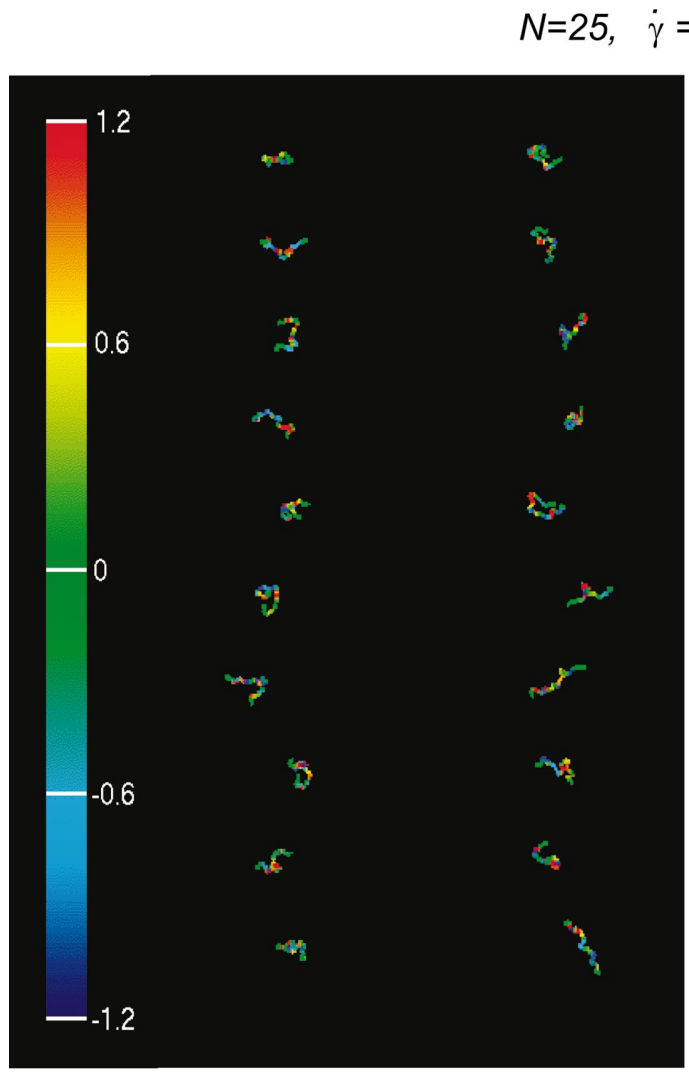

(a) $\dot{\gamma} t=0$

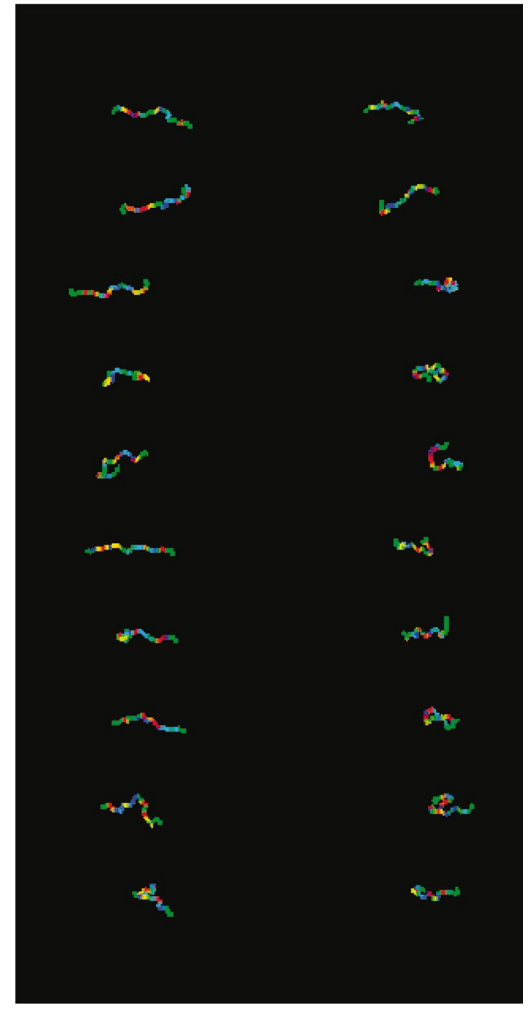

(b) $\dot{\gamma} t=10$
FIG. 8. (Color) Snapshots of short chains with $N=25$ in the $x z$ plane at $\dot{\gamma} t=5$ in (a) and at $\dot{\gamma} t$ $=10$ in (b), where a shear flow with $\dot{\gamma}=10^{-2}$ was applied at $t=0$. along the chains. In (b), on the other hand, several consecutive beads form "active spots" having relatively large values of $W_{i}(t, \tau)$. Here a bead (being the $i$ th one in a chain) is defined to be active if the average

$$
\bar{W}_{i}(t, \tau)=\frac{1}{7} \sum_{j=i-3}^{i+3} W_{j}(t, \tau)
$$

over the seven adjacent values $(j=i-3, i-2, \cdots, i+3)$ in the same chain is larger than $1.1 \sigma(\tau)$. This averaging "in space" furthermore eliminates random, small-scale fluctuations consisting of a few beads and, as a result, the variance of the average $\bar{W}_{i}(t, \tau)$ becomes $0.65 \sigma(\tau)$ for $\tau=5 \times 10^{3}$. Then we select $4 \%$ of the total beads as active ones. In Fig. 5(b) they form the active spots numbered from 1 to 18 , consisting of several consecutive active beads. In Fig. 5(c) we show $\bar{W}_{i}(t, \tau)$ for the three chains with the active spots from 4 to 7. Here we are expecting that these active spots should arise from entanglements in most cases except for accidental enhancement of the nonbonded interactions. Figure 5(b) indicates the existence of two or three entanglements on each chain leading to the estimation $N_{e} \cong 100$.

To examine the correlations in $W_{i}(t, \tau)$ along the chain contour quantitatively, we define the intrachain correlation function of the nonbonded interaction

$$
E(n, \tau)=\frac{1}{\mathcal{N}(\tau)} \sum_{j-i=n}\left[W_{i}(t, \tau)-\langle W\rangle\right]\left[W_{j}(t, \tau)-\langle W\rangle\right],
$$

where the two beads $i$ and $j$ are separated by $n$ on the same chain and the average over all the chains is taken. The normalization factor $\mathcal{N}(\tau)$ is defined such that $E(0, \tau)=1$; then, $\mathcal{N}(\tau)=M N \sigma(\tau)^{2} \propto \tau$. In Fig. 6, we show $E(n, \tau)$ in (a) and its Fourier transformation,

$$
P(k, \tau)=\sum_{n=0}^{N-1} E(n, \tau) \cos (k n),
$$

in (b) for $\tau=0,50,500,5 \times 10^{3}$, and $5 \times 10^{4}$. The longest $\tau$ is of the same order as $\tau_{e}$ in Eq. (23). The most conspicuous feature is that $E(n, \tau)$ takes a negative minimum around $n$ $=45$ and positive maxima around $n=80$ with increasing $\tau$. The average displacement $\ell(\tau)$ in Eq. (22) was calculated to be 4.5 for $\tau=5 \times 10^{3}$ and 7.7 for $\tau=5 \times 10^{4}$. The contributions giving rise to these extrema grow in time in the normalized correlation $E(n, \tau)$ or equivalently decay slower than $\tau^{-1}$ in the unnormalized correlation $E(n, \tau) \mathcal{N}(\tau)$. Correspondingly, the Fourier transformation $P(k, \tau)$ has a peak at $k$ $\cong 2 \pi / 90$ at large $\tau$. This suggests $N_{e} \cong 90$. In addition, if $\tau$ is much larger than $\tau_{e}$, no periodic structure was observed in $E(n, \tau)$ (not shown here). This should be because the entanglements are delocalized along the chains in long time intervals with $\tau \gg \tau_{e}$. Furthermore, as can be seen in the inset of Fig. 6(a), the correlations between nearby beads $E(n, \tau)$ 

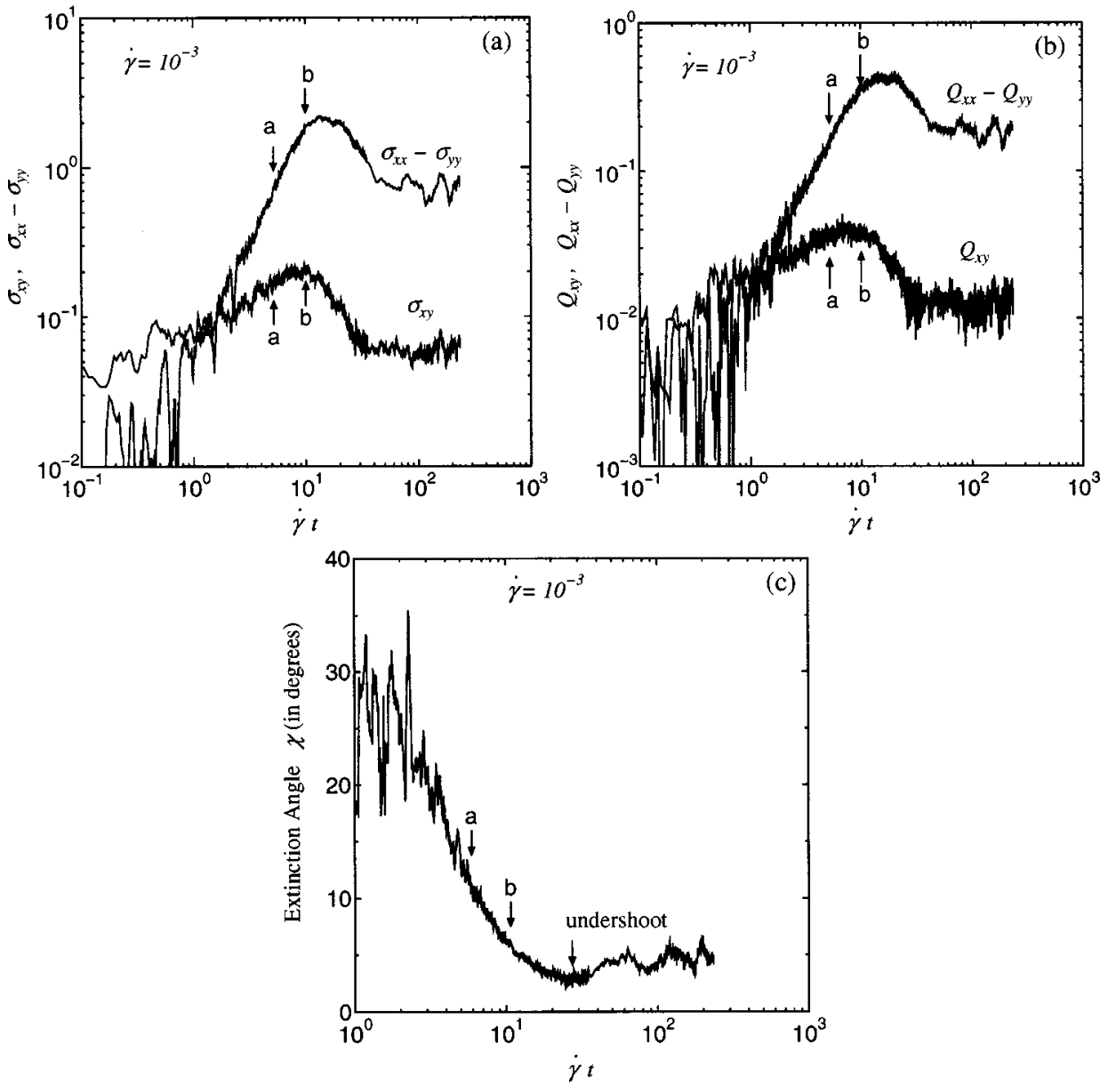

FIG. 9. The stress growth functions after application of shear flow with $\dot{\gamma}=10^{-3}$ for $N$ $=250$ in (a). The arrows (a) and (b) indicate the points of $\dot{\gamma} t=5$ in Fig. 7(a) and $\dot{\gamma} t=10$ in Fig. 7(b). The maximum of $\sigma_{x y}(t)$ is at $\dot{\gamma} t$ $=10$ and that of $N_{1}(t)$ is at $\dot{\gamma} t=20$. The corresponding components of the orientational tensor $Q_{\alpha \beta}(t)$ in Eq. (27) are shown in (b). The extinction angle $\chi$ in Eq. (30) is shown in (c), which undershoots before reaching a larger steadystate value. These figures are the results of the single run displayed in Fig. 7. with $1 \leqslant n \lesssim 10$ are nearly zero for $\tau=0$ but increases with increasing $\tau$. We may determine the characteristic width $n_{w}(\tau)$ by $E(n, \tau)>10^{-2}$ for $n<n_{w}(\tau)$. If this definition is used, the calculated values of $n_{w}(\tau)$ and $\ell(\tau)$ in Eq. (22) nearly coincide (within a few $10 \%$ ).

\section{Entanglements under rapid shearing and stress overshoot}

Next, we applied a shear flow with rate $\dot{\gamma}=10^{-3}$ $\sim 600 / \tau_{r} \sim 170 / \tau_{R}$ to the system of $N=250$ and $M=10$. We used the same initial values for the particle positions and momenta as those which produced the data shown in Fig. 5(b). This is convenient to examine how entanglements behave in the quiescent and sheared conditions starting at exactly the same conditions. Here the time scale of the flowinduced chain deformations $\left(\sim \dot{\gamma}^{-1}\right)$ is much shorter than $\tau_{e}$ in Eq. (23). In Fig. 7 the chain conformations are projected onto the $x z$ plane (perpendicular to the velocity-gradient direction) at $\dot{\gamma} t=5$ in (a) and $\dot{\gamma} t=10$ in (b). In (b) the shear stress takes a maximum as will be shown in Fig. 9. Because the chains are rapidly elongated, they eventually take zigzag shapes bent presumably at entanglements. The nonbonded interactions in these zig-zag points become increasingly amplified with increasing strain. This should be because a considerable fraction of the stress is supported by entanglements in strong deformations. As a result, active spots in $W_{i}(t, \tau)$ can be detected even with much smaller $\tau$ than in the quies- cent case, so we set $\tau=500=0.5 / \dot{\gamma}$ at this shear rate. In our simulation the noise effect in $W_{i}(t, \tau)$ is much more reduced for $\tau \sim \dot{\gamma}^{-1}$ than in the quiescent case. Roughly speaking, a $2 / 3$ fraction of the numbered active spots without shear in Fig. 5(b) remain to be active spots under shear strain of 0.5 , and a $1 / 2$ fraction of them become bent under shear in Fig. 7(a) and Fig. 7(b). As in the criterion in Fig. 5, the definition of the active beads is given by $\bar{W}_{i}(t, \tau)>1.1 \sigma(\tau)$ (but with much smaller $\tau$ ) and the number of the active beads is $4 \%$ of the total bed number. We assign the same numbers to these active spots if their contour distance between the locations along the chain in the quiescent and sheared cases remains shorter than 10. The bend regions marked by + in Fig. 7, however, do not correspond to the numbered hot spots in Fig. 5.

We can also see that the number of the bends has not decreased from Fig. 7(a) to Fig. 7(b), but several of them are approaching the chain ends and will disappear (not shown here). Notice that the shear stress is maximum at the time of Fig. 7(b). In the reptation theory it is assumed that entanglements can be released only when they reach a chain end. If our bends represent entanglements, the disentanglement process induced by shear flow is going to start in Fig. 7(b), then leading to a decrease of the shear stress.

For comparison, in Fig. 8 we show snapshots of the chains for the shorter chain case of $N=25$ in a quiescent state in (a) and under shear $\dot{\gamma}=10^{-2}$ in (b), where $\tau_{r}=1850\left(\cong \tau_{R}\right)$ 


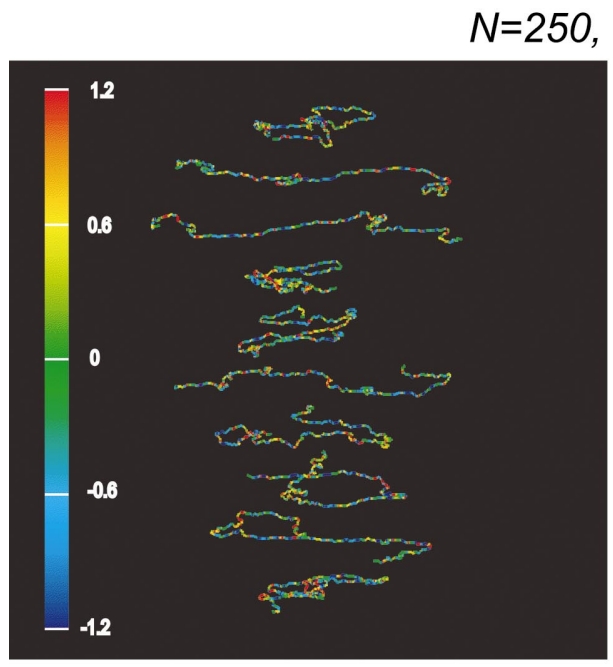

(a) $\dot{\gamma} t=5$
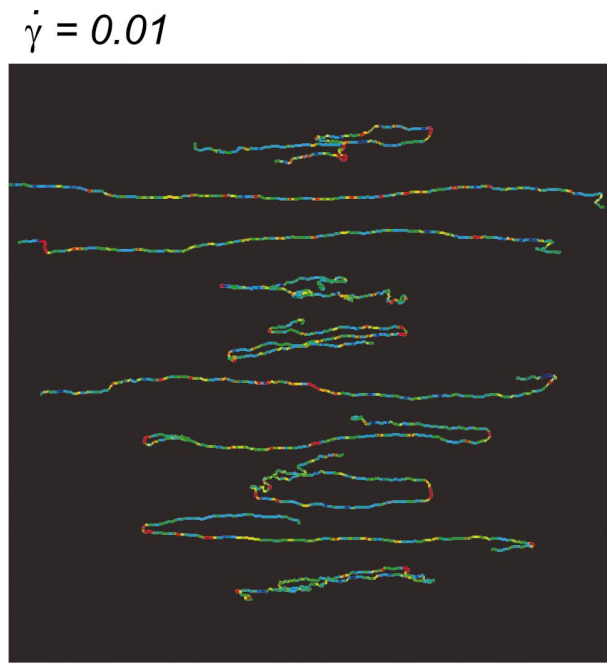

(b) $\dot{\gamma} t=12.5$
FIG. 10. (Color) Snapshots of the deformed chains with $N=250$ in the $x z$ plane at $\dot{\gamma} t=5$ in (a) and at $\dot{\gamma} t=12.5$ in (b), where a shear flow with $\dot{\gamma}=10^{-2}$ was applied at $t=0$. The nonbonded interactions with $\tau=50$ are written on the chains using the color map on the left. In (b) the stretching is nearly complete between the bends. and there is no entanglement. The nonbonded interactions are written with $\tau=50$, but we cannot see any particular meaning in its heterogeneities on the chains with and without shear. In strong shear flow $\dot{\gamma} \tau_{R} \gg 1$, the chains are at most times elongated along the flow but undergo random tumbling motions with period much longer than $\dot{\gamma}^{-1}$. In (b) compactly shaped chains are in the course of tumbling [24].

In the case of entangled melts, the shear stress $\sigma_{x y}(t)$ and the normal stress $N_{1}(t)=\sigma_{x x}(t)-\sigma_{y y}(t)$ are known to exhibit overshoot behavior in rapid shearing $[6,7,16,17,19,28]$. In Fig. 9(a) we show their time evolution after application of shear at $t=0$ with $\dot{\gamma}=10^{-3}$ in the run of Fig. 7. On the one hand, $\sigma_{x y}(t)$ takes a maximum at $\dot{\gamma} t=10$, at which the disentanglement starts. On the other hand, $N_{1}(t)$ takes a maximum at $\dot{\gamma} t=20$ afterwards. The same tendency of successive maxima of $\sigma_{x y}(t)$ and $N_{1}(t)$ was predicted theoretically [7] and observed experimentally under rapid shearing [6,27,28]. In Fig. 9(b) we show the numerical result of the orientational tensor $Q_{\alpha \beta}(t)$ defined by

$$
Q_{\alpha \beta}(t)=\frac{1}{M} \sum_{\text {chain }} \frac{1}{N-1} \sum_{j=1}^{N-1} b_{\min }^{-2} b_{j \alpha}(t) b_{j \beta}(t),
$$

where $b_{\min }^{-1} \mathbf{b}_{j}(t)$ are the normalized bond vectors and $\Sigma_{\alpha} Q_{\alpha \alpha} \cong 1$ since $\left|\mathbf{b}_{j}\right| \cong b_{\text {min }} \cong 0.96 \sigma$ as stated below Eq. (2). For this shear rate $\left(\dot{\gamma}=10^{-3}\right), Q_{x x}(t)-Q_{y y}(t)$ remains considerably smaller than 1 and the chain bonds are still weakly oriented along the flow direction. The overshoot of $Q_{x x}(t)$ $-Q_{y y}(t)$ indicates retraction of the chain contours (tubes) after onset of disentanglement. Comparing Fig. 9(a) and 9(b), we notice the proportionality of the two deviatoric components of the two tensors

$$
\sigma_{x y}(t)=A_{0} Q_{x y}(t), \quad N_{1}(t)=A_{0}\left[Q_{x x}(t)-Q_{y y}(t)\right],
$$

where $A_{0}=2.2$ (with the stress components being measured in units of $\epsilon \sigma^{-3}$ ) [38]. Note that the deviatoric part of the stress of polymer melts is believed to be nearly equal to that of the entropic stress contribution $\left(\sim k_{B} \operatorname{Tn} Q_{\alpha \beta}\right)$ far above the glass transition temperature [2,3]. Furthermore, the devia- toric part of the dielectric tensor $\epsilon_{\alpha \beta}$ is proportional to that of $Q_{\alpha \beta}$ provided that the microscopic polarization tensor is uniaxial along the bond direction. Thus we obtain the wellknown stress-optical relations

$$
\epsilon_{x y}=C_{0} \sigma_{x y}, \quad \epsilon_{x x}-\epsilon_{y y}=C_{0}\left(\sigma_{x x}-\sigma_{y y}\right)
$$

where $C_{0}$ is a polymer-dependent constant. In Fig. 9(c) we also show the following angle:

$$
\chi=\frac{1}{2} \tan ^{-1}\left(2 \sigma_{x y} / N_{1}\right)
$$

On the basis of the stress-optical law, this angle is measured as the extinction angle $\chi=(1 / 2) \tan ^{-1}\left[2 \epsilon_{x y} /\left(\epsilon_{x x}-\epsilon_{y y}\right)\right]$ in birefringence experiments. In Fig. 8(c) we can see that $\chi$ exhibits a small undershoot around $\dot{\gamma} t=30$ after the peaks of $\sigma_{x y}(t)$ and $N_{1}(t)$. A similar retarded undershoot was observed experimentally but has not been explained theoretically [7].

As the final example, we examine the case of much larger shear rate $\dot{\gamma}=10^{-2} \sim 6000 / \tau_{r} \sim 1700 / \tau_{R}$. Figure 10 displays the snapshots of the chain conformations for $\dot{\gamma} t=5$ in (a) and for $\dot{\gamma} t=12.5$ in (b), where the chain stretching is stronger than in Fig. 7 and the numbers of entanglements remain unchanged. The time interval $\tau$ in Eq. (19) is set equal to 50. In Fig. 11 we show $\sigma_{x y}(t)$ and $N_{1}(t)$ in (a), both exhibiting a peak around $\dot{\gamma} t \cong 12.5$, and $Q_{x y}(t)$ and $Q_{x x}(t)-Q_{y y}(t)$ in (b). The stress overshoot is more enhanced than in the smaller shear case in Fig. 9, and the stress components decrease rather abruptly with onset of disentanglement. However, $Q_{x x}(t)-Q_{y y}(t)$ saturates to a value about 0.7 without exhibiting overshoot. Here even the bonds themselves align in the flow direction and the chain stretching becomes nearly complete. Interestingly, this bond alignment is still maintained even after onset of disentanglement. As we remarked below Eq. (2), bond elongation of order $3 \%$ is anharmonic for the potentials in Eq. (1) and (2) and gives rise to a tensile force of order $\epsilon / \sigma$. In this simulation, such strong forces are exerted on most of the bonds and the proportionality relation in Eq. (28) does not hold. 

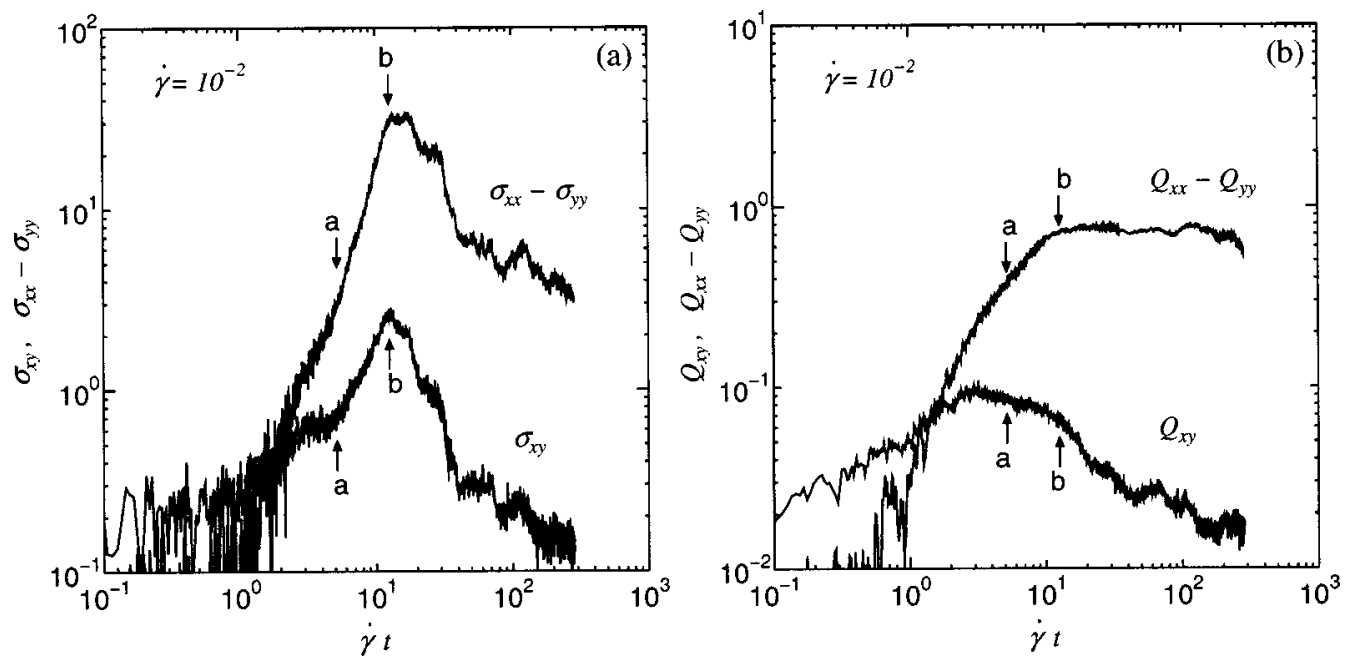

FIG. 11. The stress growth functions after application of shear flow with $\dot{\gamma}=10^{-2}$ for $N=250$ in (a). The arrows (a) and (b) indicate the points of $\dot{\gamma} t=5$ in Fig. 10(a) and $\dot{\gamma} t=12.5$ in Fig. 10(b). The corresponding components of the orientational tensor $Q_{\alpha \beta}(t)$ are shown in (b), which demonstrate bond elongation along the flow and behave very differently from the stress components.

\section{SUMMARY AND CONCLUDING REMARKS}

We have presented attempts to detect and visualize the entanglements in a model polymer melt together with attempts of indirectly deriving $N_{e}$ as in the previous simulations. All the methods have yielded $N_{e} \sim 100$. We admit that the visualization in the quiescent case is not yet firmly established by themselves in view of the fact that the thermal noise still affects the data even after averaging in space and time as in Fig. 5(c). However, under rapid shearing, a large fraction of active spots with relatively large nonbonded interactions become bent, evidently indicating the existence of obstacles for the chain motion. Remarkably, the active spots in the quiescent and sheared cases in Fig. 5 and 7 coincide with a large probability $(\sim 2 / 3)$. We claim that a large fraction of such obstacles arise from entanglements preexisting even before application of shear. However, a few bends and hot spots in Fig. 7 are not detected by the visualization method in Fig. 5. This suggests that some hot spots from our method may not represent entanglements.

We have detected discrete obstacles in the chains. In the future we should examine how they are related to the concepts of tubes in the reptation theory [2-5,11]. We mention the work by Everaers et al., which numerically detected the primitive paths [8].

As remarked already at the end of Sec. II A, the system size in our simulations is still comparable to the end-to-end distance for $N=250$ and our results need to be further checked in future large-scale simulations with longer chains. To eliminate the large thermal noise effect in the quiescent case, we should take data in the well-defined reptation regime under $N \gg N_{e}$.
Performing very long simulations, we have also calculated the stress relaxation function $G(t)$, which exhibits the Rouse-to-reptation crossover with increasing the polymerization index $N$, and studied nonlinear rheology in transient and steady sheared states. In transient states under shear, the stress overshoot sets in as the bends approach the chain ends and disappear, as can be seen from Fig. 7(b) and 9. This is also one of our main results giving molecular information on the stress overshoot under rapid shearing.

In real long chain systems, the ratio $\tau_{d} / \tau_{R} \sim N / N_{e}$ can be very large. Hence, in shear flow, there can be three characteristic shear regions [7] given by (i) $\dot{\gamma}<\tau_{d}^{-1}$, (ii) $\tau_{d}^{-1}<\dot{\gamma}<\tau_{R}^{-1}$, and (iii) $\dot{\gamma}>\tau_{R}^{-1}$. Nonlinear shear effects emerge in the regions (ii) and (iii), while the linear response theory in terms of $G(t)$ in Eq. (11) is valid only in the region (i). In our study, the intermediate region (ii) is not welldefined, but the calculated overshoot and undershoot relaxations in Fig. 9 [in the region (iii)] resemble those in the experiments $[6,27,28]$.

\section{ACKNOWLEDGMENTS}

This work was supported by Grants in Aid for Scientific Research and by the 21st Century COE project (Center for Diversity and Universality in Physics) from the Ministry of Education, Culture, Sports, Science and Technology of Japan. Calculations were performed at the Human Genome Center, Institute of Medical Science, University of Tokyo and the Supercomputer Center, Institute for Solid State Physics, University of Tokyo. 
[1] J. D. Ferry, Viscoelastic Properties of Polymers, 3rd ed. (Wiley, New York, 1983).

[2] P. G. de Gennes, Scaling Concepts in Polymer Physics (Cornell University Press, Ithaca, 1979).

[3] M. Doi and S. F. Edwards, The Theory of Polymer Dynamics (Clarendon, Oxford, 1986).

[4] T. C. B. McLeish, Adv. Phys. 51, 1379 (2002).

[5] M. Rubinstein and R. H. Colby, Polymer Physics (Oxford University Press, Oxford, 2003); M. Rubinstein, in Theoretical Challenges in the Dynamics of Complex Fluids, edited by T. C. B. McLeish (Kluwer Academic, Dordrecht, 1997).

[6] D. S. Pearson, E. Herbolzheimer, N. Grizzuti, and G. J. Marrucci, J. Polym. Sci., Part B: Polym. Phys. 29, 1589 (1991).

[7] D. W. Mead, R. G. Larson, and M. Doi, Macromolecules 31, 7895 (1998).

[8] R. Everaers, S. K. Sukumaran, G. S. Grest, C. Svaneborg, A. Sivasubramanian, and K. Kremer, Science 303, 823 (2004).

[9] D. Richter, R. Butera, L. J. Fetters, J. S. Huang, B. Farago, and B. Ewen, Macromolecules 23, 6156 (1992); P. Schleger, B. Farago, C. Lartigue, A. Kollmar, and D. Richter, Phys. Rev. Lett. 81, 124 (1998).

[10] U. Ebert, A. Baumgärtner, and L. Schäfer, Phys. Rev. Lett. 78, 1592 (1997).

[11] K. Kremer and G. S. Grest, J. Chem. Phys. 92, 5057 (1990).

[12] W. Paul, K. Binder, D. W. Heermann, and K. Kremer, J. Chem. Phys. 95, 7726 (1991).

[13] S. W. Smith, C. K. Hall, and B. D. Freeman, Phys. Rev. Lett. 75, 1316 (1995); J. Chem. Phys. 104, 5616 (1996).

[14] M. Pütz, K. Kremer, and G. S. Grest, Europhys. Lett. 49, 735 (2000).

[15] J. Gao and J. H. Weiner, J. Chem. Phys. 103, 1614 (1995); 103, 1621 (1995).

[16] M. Kröger, W. Loose, and S. Hess, J. Rheol. 37, 1057 (1993).

[17] S. Hess, C. Aust, L. Bennet, M. Kröger, C. P. Borgmeyer, and T. Weider, Physica A 240, 126 (1997).

[18] M. Kröger, and S. Hess, Phys. Rev. Lett. 85, 1128 (2000).

[19] T. Aoyagi and M. Doi, Comput. Theor. Polym. Sci. 10, 317 (2000).

[20] J. T. Padding and W. J. Briels, J. Chem. Phys. 114, 8685 (2001).

[21] J. T. Padding and W. J. Briels, J. Chem. Phys. 117, 925 (2002).

[22] R. G. Larson, T. Sridhar, L. G. Leal, G. H. McKinley, A. E.
Likhtman, and T. C. B. McLeish, J. Rheol. 47, 809 (2003).

[23] J. D. Moore, S. T. Cui, H. D. Cochran, and P. T. Cummings, J. Non-Newtonian Fluid Mech. 93, 83 (2000); 93, 101 (2000).

[24] R. Yamamoto and A. Onuki, J. Phys.: Condens. Matter 29, 6323 (2000); J. Chem. Phys. 117, 2359 (2002).

[25] If a chain system is near the glass transition, the stress relaxation becomes extremely slow and nonlinear shear regimes are realized even at very small shear rates. See calculations of $G(t)$ and rheology in such a system [24,33].

[26] E. Ben-Naim, G. S. Grest, T. A. Witten, and A. R.C. Baljon, Phys. Rev. E 53, 1816 (1996).

[27] E. V. Menezes and W. W. Graessley, J. Polym. Sci., Polym. Phys. Ed. 20, 1817 (1982).

[28] T. Inoue, Y. Yamashita, and K. Osaki, Macromolecules 35, 1770 (2002).

[29] M. P. Allen and D. J. Tildesley, Computer Simulation of Liquids (Clarendon, Oxford, 1987).

[30] D. J. Evans and G. P. Morriss, Statistical Mechanics of Nonequilibrium Liquids (Academic, New York, 1990).

[31] For example, the fluctuation of $\left\langle\left|\mathbf{P}\left(t_{0}\right)\right|^{2}\right\rangle$ as a function of $t_{0}$ was of order $6 \%$ for $N=10$ and $M=100$ and was of order $25 \%$ for $N=250$ and $M=10$, as compared to the time averages over a time interval of $10^{6} \tau_{0}$.

[32] If $b$ is determined by Eq. (4), it becomes very weakly dependent on $N$ as $b=1.04(N-1)^{0.05}$ in our simulations.

[33] A. Onuki, Phase Transition Dynamics (Cambridge University Press, Cambridge, 2002).

[34] R. Yamamoto and Y. Mori (unpublished). We have performed simulations using a model with fixed bond lengths, for which the initial oscillatory behavior of $G(t)$ becomes nonexistent.

[35] R. A. Stratton, J. Colloid Interface Sci. 22, 517 (1966).

[36] D. Thirumalai and R. D. Mountain, Phys. Rev. E 47, 479 (1993).

[37] There is large ambiguity in the prefactor of $\tau_{e}$ [9] . That is, $\tau_{e}=(\pi / 12) \zeta b^{2} N_{e}^{2} / k_{B} T$ if $\ell\left(\tau_{e}\right)=a=N_{e}^{1 / 2} b$ [3]. However, $\tau_{e}$ $=\left(1 / 3 \pi^{2}\right) \zeta b^{2} N_{e}^{2} / k_{B} T$ if $\tau_{e}=\tau_{R}$ for $N=N_{e}$.

[38] In Ref. [24], we used the same chain model with $N=10$ and $n \sigma^{3}=1$ to obtain $A_{0}=2.2 k_{B} T n$ for various $T$ as the proportionality coefficient of the relation in Eq. (28). See Fig. 6 of Ref. [24]. This expression also holds in the present work and is thus independent of $N$. 